\title{
Sustainable Supply Chain Network Design: A Multicriteria Perspective
}

\author{
Anna Nagurney \\ Department of Finance and Operations Management \\ Isenberg School of Management \\ University of Massachusetts \\ Amherst, Massachusetts 01003 \\ Ladimer S. Nagurney \\ Department of Electrical and Computer Engineering \\ University of Hartford \\ West Hartford, Connecticut 06117
}

August 2009; revised April 2010

International Journal of Sustainable Engineering 3 (2010): pp. 189-197.

\begin{abstract}
In this paper we develop a rigorous modeling and analytical framework for the design of sustainable supply chain networks. We consider a firm that is engaged in determining the capacities of its various supply chain activities, that is, the manufacturing, storage, and distribution of the product to the demand locations. The firm is faced with both capital costs associated with constructing the link capacities as well as the links' operational costs. Moreover, the firm is aware of the emissions generated associated with the alternative manufacturing plants, storage facilities, and modes of transportation/shipment, which may have different levels of emissions due, for example, to distinct technologies of, respectively, production, storage, and transportation. The firm is assumed to be a multicriteria decisionmaker who seeks to not only minimize the total costs associated with design/construction and operation, but also to minimize the emissions generated, with an appropriate weight, which reflects the price of the emissions, associated with the various supply chain network activities. We provide both the network optimization modeling framework and an algorithm, which is then applied to compute solutions to a spectrum of numerical sustainable supply chain design examples in order to illustrate our approach.
\end{abstract}

Keywords: supply chains, sustainability, network design, multicriteria decision-making, optimization 


\section{Introduction}

Supply chain networks provide the infrastructure for the production, storage, and distribution of products as varied as pharmaceuticals, vehicles, computers, food products, furniture, and clothing, throughout the globe. Hence, the design of supply chain networks is a topic of engineering importance since it involves the determination of both the sites and the levels of operation of the relevant facilities that enable the manufacture, storage, and delivery of products to the consumers. Simultaneously, sustainability of supply chains has emerged as a major theme in both research and practice since the impacts of climate change have made both producers and consumers more cognizant of their decision-making and how their decisions affect the environment.

In a series of papers (cf. Nagurney, Cruz, and Matsypura (2003), Nagurney and Toyasaki (2005), Wu et al. (2006), Nagurney, Liu, and Woolley (2007), Nagurney and Woolley (2010)), it has been argued that businesses, and in particular supply chains, have become increasingly globalized. However, criticism of globalization has increased, specifically by those concerned about the environment on the basis that global free trade may result in the growth of global pollution. For example, free trade may shift pollution-intensive manufacturing processes from countries with strict environmental regulations to those with less restrictive ones. Nevertheless, legal requirements and evolving consumer tastes are placing pressure on manufacturers and distributors to become more environmentally-friendly and to minimize the emissions generated (cf. Bloemhof-Ruwaard et al. (1995), Hill (1997), and Ingram (2002)). Indeed, as noted in Nagurney (2006), firms are being held accountable not only for their own environmental performance, but also for that of their suppliers, distributors, and even, ultimately, for the environmental consequences of the disposal of their products. Poor environmental performance at any stage of the supply chain process may, thus, damage what is considered a firm's premier asset, its reputation (see Fabian (2000)).

In this paper, we develop a multicriteria perspective for sustainable supply chain network design. The mathematical model that we propose allows for the simultaneous determination of supply chain network link capacities, through capital investments, and the product flows on various links, that is, the manufacturing, storage, distribution/shipment links, etc., coupled with the emissions generated. The total cost associated with emission-generation 
consists of the price per unit of emission times the volume of the emissions (with the values being possibly distinct for each link). Specifically, the optimization model that we develop guarantees that the demands for the product are satisfied at minimal total cost, where the objective function also includes the total cost associated with environmental emissions. Our model for sustainable supply chain network design, as we demonstrate, captures, in a graphical manner, the options available, and provides flexibility in terms of the evaluation of trade-offs of the where and the how of production, storage, and distribution of the product and the associated environmental impacts. Additional background on sustainable design and manufacturing can be found in Rahimifard and Clegg (2007).

Optimization models have been developed for supply chain network integration in the case of mergers and acquisitions that also capture potential environmental synergies associated with supply chain network integration (Nagurney and Woolley (2010)). However, in those models, in contrast to the one in this paper, it is assumed that the capacities on the supply chain network links are fixed and known. An alternative approach to supply chain networks (cf. Nagurney, Dong, and Zhang (2002)) considers competition among decision-makers in supply chains and uses equilibrium (as opposed to optimization) as the governing concept. In such supply chain network equilibrium models (see also Qiang, Nagurney, and Dong (2009), and the references therein) there are no explicit capacity link variables. The design issue in such models is, typically, handled by eliminating the links in the solution that have zero product flows.

In the model in this paper, in contrast to those referenced above, the capacities are design decision variables. The novelty of this approach also lies in that we utilize continuous variables exclusively as decision variables. When the optimal solution results in zero capacities associated with particular links, then those links can, in effect, be removed from the final optimal supply chain network design. This does not limit the generality of the approach; rather, it adds flexibility and the ability to handle large-scale design problems plus it allows for the application of an effective algorithm that exploits the network structure of the problem.

This paper is organized as follows. In Section 2, we develop the sustainable supply chain network design model, in which capacity levels and the product flows are endogenous 
variables. The firm is a multicriteria decision-maker and seeks to minimize the total costs and to minimize the total emissions generated, with an associated weight. We establish that the optimization problem is equivalent to a variational inequality problem, with nice features for computations. The solution of the sustainable supply chain network design model yields the optimal capacities and product flows of the supply chain network, so that the total cost, which includes the weighted emissions generated, is minimized and the demands are satisfied. We also propose an algorithm, which exploits the underlying structure of the problem, and which computes the optimal capacities, the product flows, and also the relevant Lagrange multipliers. In addition, we establish convergence of the algorithm for the solution of our model. In Section 3 we apply the algorithm to several numerical sustainable supply chain network design examples. In Section 4, we summarize the results in this paper and present our conclusions. 


\section{The Sustainable Supply Chain Network Design Model}

In this Section, we develop the sustainable supply chain network design model. We assume that the firm responsible for ensuring that the demand for the product be met is considering its possible supply chain activities, associated with the product, which are represented by a network topology. For clarity and definiteness, we consider the network topology depicted in Figure 1 but emphasize that the modeling framework developed here is not limited to such a network. Indeed, as will become apparent, what is required, to begin with, is the appropriate network topology with a top level (origin) node 0 corresponding to the firm and the bottom level (destination) nodes corresponding to the demand sites, which can correspond, for example, to retailers or consumers, that the firm wishes to supply. The paths joining the origin node to the destination nodes represent sequences of supply chain network activities that ensure that the product is produced and, ultimately, delivered to the demand sites.

We assume that in the supply chain network topology there exists at least one path joining node 0 with each destination node. This assumption for the supply chain network design model guarantees that the demand at each demand point will be met. The solution of the model will then yield the optimal product flows and capacity investments at minimum total cost and the minimum total emissions (with appropriate firm-imposed weights). Note that the supply chain network schematic, as in Figure 1, provides the foundation upon which the optimal supply chain network design will be determined.

In particular, as depicted in Figure 1, we assume that the firm is considering $n_{M}$ manufacturing facilities/plants; $n_{D}$ distribution centers, and is to serve the $n$ demand locations with the respective demands given by: $d_{1}, d_{2}, \ldots, d_{n}$. The links from the top-tiered node 0 are connected to the possible manufacturing nodes of the firm, which are denoted, respectively, by: $M_{1}, \ldots, M_{n_{M}}$, and these links represent the manufacturing links. Note that, as depicted in Figure 1, there may be multiple alternative links joining node 0 to one of the manufacturing nodes. These links correspond to different possible technologies associated with a given manufacturing plant, which, as we shall see below may also result in different levels of environmental emissions. For example, a firm in deciding upon its mix of manufacturing plants may also select the underlying technology for the manufacturing processes, 
which will, in turn, be associated with a particular level of emissions.

The links from the manufacturing nodes, in turn, are connected to the possible distribution center nodes of the firm, and are denoted by $D_{1,1}, \ldots, D_{n_{D}, 1}$. These links correspond to the possible shipment links between the manufacturing plants and the distribution centers where the product will be stored. The links joining nodes $D_{1,1}, \ldots, D_{n_{D}, 1}$ with nodes $D_{1,2}, \ldots, D_{n_{D}, 2}$ correspond to the possible storage links. Finally, there are possible shipment links joining the nodes $D_{1,2}, \ldots, D_{n_{D}, 2}$ with the demand nodes: $1, \ldots, n$. There may also exist multiple possibilities of transportation and storage, reflecting, as in the case of manufacturing, different technologies and associated emissions generated. Note that in Figure 1 such alternatives are depicted as distinct links joining a pair of nodes.

We denote the supply chain network consisting of the graph $G=[N, L]$, where $N$ denotes the set of nodes and $L$ the set of directed links. Note that $G$ represents the topology of the full supply chain network possibilities (as in Figure 1, for example). The ultimate solution of the complete model will yield the optimal sustainable supply chain network design (which may only have a subset of the links in Figure 1).

As mentioned in the Introduction, the formalism that we utilize is that of optimization, where the firm wishes to determine which manufacturing plants it should operate (and the corresponding technologies) and at what level; the same for the distribution centers. In addition, the firm seeks to determine the capacity levels of the shipment links and the mode of transportation/shipment used (which may be more or less polluting). We assume that the firm seeks to minimize the total costs associated with its production, storage, distribution activities, along with the total capital outlays, plus the total cost of environmental emissions, which we elaborate upon below, in order to achieve the activity levels as given by the capacities on its various links, subject to the demand being satisfied at the demand sites. 


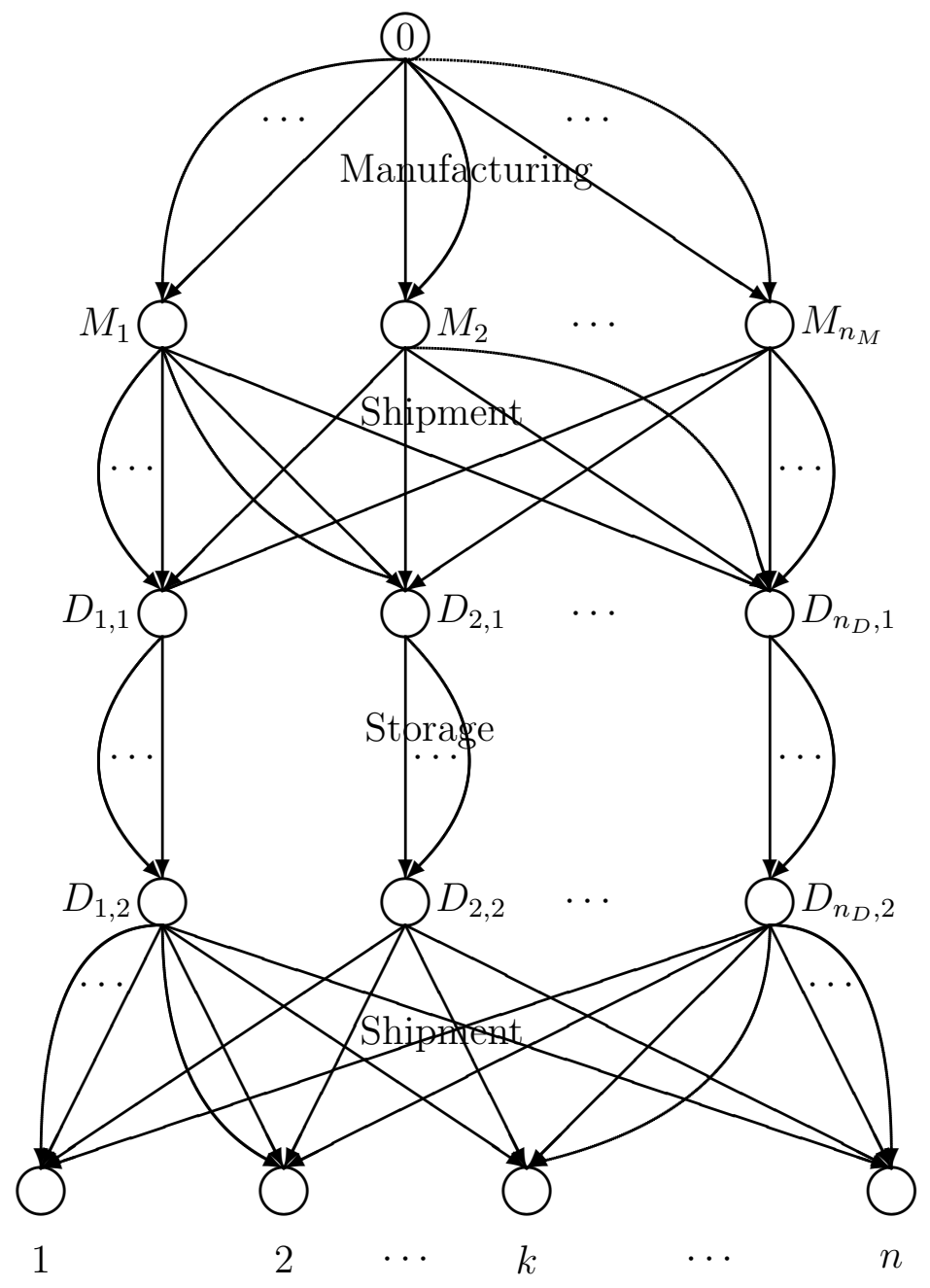

Figure 1: The Baseline Supply Chain Network Topology 
Associated with each link (cf. Figure 1) of the network is a total cost that reflects the total cost of operating the particular supply chain activity, that is, the manufacturing of the product, the shipment of the product, the storage of the product, etc., over the time horizon underlying the design problem. We denote, without any loss in generality, the links by $a, b$, etc., and the total cost on a link $a$ by $\hat{c}_{a}$. For the sake of generality, we note that the total costs are generalized costs and may include, for example, risk, time, etc.

A path $p$ in the network (see, e.g., Figure 1) joining node 0, which is the origin node, to a demand node, which is a destination node, represents the activities and their sequence associated with producing the product and having it, ultimately, delivered. Let $w_{k}$ denote the pair of origin/destination $(\mathrm{O} / \mathrm{D})$ nodes $(0, k)$ and let $P_{w_{k}}$ denote the set of paths, which represent alternative associated possible supply chain network processes, joining $(0, k) . P$ then denotes the set of all paths joining node 0 to the demand nodes. Let $n_{P}$ denote the number of paths from the organization to the demand markets.

Let $x_{p}$ represent the nonnegative flow of the product on path $p$ joining (origin) node 0 with a (destination) demand node that the firm is to supply with the product.

Let $d_{k}$ denote the demand, which is assumed to be known and fixed, for the product at demand location $k$. Then, the following conservation of flow equation must hold:

$$
d_{k} \equiv \sum_{p \in P_{w_{k}}} x_{p}, \quad k=1, \ldots, n
$$

that is, the demand must be satisfied at each demand site.

In addition, let $f_{a}$ denote the flow of the product on link $a$. Hence, we must have the following conservation of flow equations satisfied:

$$
f_{a}=\sum_{p \in P} x_{p} \delta_{a p}, \quad \forall a \in L
$$

where $\delta_{a p}=1$, if link $a$ is contained in path $p$, and $\delta_{a p}=0$, otherwise; that is, the total amount of a product on a link is equal to the sum of the flows of the product on all paths that utilize that link.

Of course, we also have that the path flows must be nonnegative, that is,

$$
x_{p} \geq 0, \quad \forall p \in P
$$


since the product will be produced in nonnegative quantities.

The total cost on a link, be it a manufacturing/production link, a shipment link, or a storage link is assumed to be a function of the flow of the product on the link; see, for example, Nagurney (2006) and the references therein. We have, thus, that

$$
\hat{c}_{a}=\hat{c}_{a}\left(f_{a}\right), \quad \forall a \in L
$$

We assume that the total cost on each link is convex and is continuously differentiable.

We denote the total capital cost of adding capacity $u_{a}$ on link $a$ by $\hat{\pi}_{a}, \forall a \in L$, and assume that

$$
\hat{\pi}_{a}=\hat{\pi}_{a}\left(u_{a}\right), \quad \forall a \in L,
$$

that is, the total cost associated with adding capacity $u_{a}$ on link $a$ is a function of the added capacity on the link. These functions are assumed to be convex and continuously differentiable and to have bounded second order partial derivatives.

The sustainable supply chain network design optimization problem faced by the firm can be expressed as follows. The firm seeks to determine the optimal levels of product processed on each supply chain network link coupled with the optimal levels of capacity investments in its supply chain network activities subject to the minimization of the total cost. The total cost includes the total cost of operating the various links and the total cost of capacity capital investments. Hence, the firm is faced with the following objective function:

$$
\text { Minimize } \sum_{a \in L} \hat{c}_{a}\left(f_{a}\right)+\hat{\pi}_{a}\left(u_{a}\right) \text {. }
$$

In addition, it is assumed that the firm is concerned with the total amount of emissions generated both in the capital phase and in the operation phase of the supply chain and the emissions generated can occur in the manufacture, storage, and shipment of the product. Letting $e_{a}\left(f_{a}\right), \forall a \in L$, denote the emission-generation function associated with link $a$ in the operation phase, which is assumed to be a function of the amount of product flow on the link. Also, let $\hat{e}_{a}\left(u_{a}\right), a \in L$, denote the emission-generation function associated with link $a$ in the capital investment phase (which corresponds to the actual "construction" of the link). 
These functions are also assumed to be convex and continuously differentiable and to have bounded second order partial derivatives. (Such assumptions are not unreasonable and are needed to establish convergence of the algorithm.) For definiteness, one may assume that the emission is a carbon emission. Examples of functional forms and references can be found in Nagurney, Qiang, and Nagurney (2010); see also Dhanda, Nagurney, and Ramanujam (1999).

The second objective of the firm is then given by:

$$
\text { Minimize } \sum_{a \in L} e_{a}\left(f_{a}\right)+\hat{e}_{a}\left(u_{a}\right) \text {. }
$$

\section{The Multicriteria Optimization Problem for Sustainable Supply Chain Network Design}

A nonnegative constant $\omega$ is now assigned to the emission-generation criterion (7). The constant $\omega$ is a weight that the firm assigns to the emission minimization criterion. This weight is, in effect, a price per unit of emissions that the firm is willing to pay. Of course, $\omega$ can also be interpreted as a "tax" imposed by the governmental/environmental authority (see, e.g., Wu et al. (2006)). Using results from multicriteria optimization (see, e.g., Nagurney and Dong (2002)), one can then construct the following objective function which combines both criteria of the firm:

$$
\text { Minimize } \sum_{a \in L} \hat{c}_{a}\left(f_{a}\right)+\hat{\pi}_{a}\left(u_{a}\right)+\omega\left(\sum_{a \in L} e_{a}\left(f_{a}\right)+\hat{e}_{a}\left(u_{a}\right)\right)
$$

The firm, hence, seeks to solve (8), subject to the constraints: (1), (2), (3), and

$$
\begin{aligned}
& f_{a} \leq u_{a}, \quad \forall a \in L, \\
& 0 \leq u_{a}, \quad \forall a \in L .
\end{aligned}
$$

Constraint (9) guarantees that the product flow on a link does not exceed that link's capacity. Constraint (10) guarantees that the link capacities are nonnegative.

We now provide the variational inequality formulation of the above multicriteria sustainable supply chain network design optimization problem. For background on variational 
inequalities, see Nagurney (1999). A variational inequality formulation will enable the solution of our design problem in an elegant and effective manner. Observe that the above optimization problem is characterized, under our assumptions, by a convex objective function and the feasible set defined by the above constraints is convex.

Clearly, the solution of the above optimization problem will yield the product flows and the link capacities that minimize the total costs and the total weighted emissions associated with the supply chain network design faced by the firm. Under the above imposed assumptions, the optimization problem is a convex optimization problem.

We associate the Lagrange multiplier $\beta_{a}$ with constraint (9) for each link $a \in L$ and we denote the associated optimal Lagrange multiplier by $\beta_{a}^{*}$. These terms may also be interpreted as the price or value of an additional unit of capacity on link $a$. We group these Lagrange multipliers into the respective vectors $\beta$ and $\beta^{*}$.

We now state the following result in which we provide variational inequality formulations of the problem in link flows.

\section{Theorem 1}

The optimization problem (8), subject to the constraints (1) - (3) and (9), (10), is equivalent to the variational inequality problem: determine the vectors of link flows, link capacities, and Lagrange multipliers $\left(f^{*}, u^{*}, \beta^{*}\right) \in K$, such that:

$$
\begin{gathered}
\sum_{a \in L}\left[\frac{\partial \hat{c}_{a}\left(f_{a}^{*}\right)}{\partial f_{a}}+\omega \frac{\partial e_{a}\left(f_{a}^{*}\right)}{\partial f_{a}}+\beta_{a}^{*}\right] \times\left[f_{a}-f_{a}^{*}\right]+\sum_{a \in L}\left[\frac{\partial \hat{\pi}_{a}\left(u_{a}^{*}\right)}{\partial u_{a}}+\omega \frac{\partial \hat{e}_{a}\left(u_{a}^{*}\right)}{\partial u_{a}}-\beta_{a}^{*}\right] \times\left[u_{a}-u_{a}^{*}\right] \\
+\sum_{a \in L}\left[u_{a}^{*}-f_{a}^{*}\right] \times\left[\beta_{a}-\beta_{a}^{*}\right] \geq 0, \quad \forall(f, u, \beta) \in K
\end{gathered}
$$

where $K \equiv\{(f, u, \beta) \mid \exists x \geq 0$, and (1), (2), (3), and (10) hold, and $\beta \geq 0\}$, where $f$ is the vector of link flows, $u$ is the vector of link capacities, and $x$ is the vector of path flows.

Proof: See Bertsekas and Tsitsiklis (1989) page 287.

Note that variational inequality (11) can be put into standard form (see Nagurney (1999)): 
determine $X^{*} \in \mathcal{K}$ such that:

$$
\left\langle F\left(X^{*}\right)^{T}, X-X^{*}\right\rangle \geq 0, \quad \forall X \in \mathcal{K}
$$

where $\langle\cdot, \cdot\rangle$ denotes the inner product in $\mathcal{N}$-dimensional Euclidean space. If we define the column vectors: $X \equiv(f, u, \beta)$ and $F(X) \equiv\left(F_{1}(X), F_{2}(X), F_{3}(X)\right)$, such that

$$
\begin{gathered}
F_{1}(X) \equiv\left[\frac{\partial \hat{c}_{a}\left(f_{a}\right)}{\partial f_{a}}+\omega \frac{\partial e_{a}\left(f_{a}\right)}{\partial f_{a}}+\beta_{a} ; \quad a \in L\right], \\
F_{2}(X) \equiv\left[\frac{\partial \hat{\pi}_{a}\left(u_{a}\right)}{\partial u_{a}}+\omega \frac{\partial \hat{e}_{a}\left(u_{a}\right)}{\partial u_{a}}-\beta_{a} ; a \in L\right], \\
F_{3}(X) \equiv\left[u_{a}-f_{a} ; \quad a \in L\right],
\end{gathered}
$$

and define $\mathcal{K} \equiv K$, then (11) can be re-expressed as (12).

In order to establish the convergence of the algorithm for our model, we first prove two theorems below. For example, variational inequality (11) can be easily solved using the modified projection method (also sometimes referred to as the extragradient method). The elegance of this computational procedure in the context of variational inequality (11) lies in that it allows one to utilize algorithms for the solution of the uncapacitated systemoptimization problem (for which numerous algorithms exist in the transportation science literature) with straightforward update procedures at each iteration to obtain the link capacities and the Lagrange multipliers. To solve the former problem we utilize in Section 3 the well-known equilibration algorithm (system-optimization version) of Dafermos and Sparrow (1969), which has been widely applied (see also, e.g., Nagurney (1999, 2006)). Recall that the modified projection method (cf. Korpelevich (1977)) is guaranteed to converge to a solution of a variational inequality problem, provided that the function that enters the variational inequality problem is monotone and Lipschitz continuous (conditions that are satisfied under the above imposed assumptions on the cost and emission functions) and that a solution exists.

Once we have solved problem (11) we have the solution $\left(f^{*}, u^{*}\right)$ that minimizes the objective function (8) associated with the design of the sustainable supply chain network.

For completeness, we now establish both monotonicity of $F(X)$ above as well as Lipschitz continuity. 


\section{Theorem 2}

The function $F(X)$ as defined following (12) (see (13) - (15)) is monotone, that is,

$$
\left\langle\left(F\left(X^{1}\right)-F\left(X^{2}\right)\right)^{T}, X^{1}-X^{2}\right\rangle \geq 0, \quad \forall X^{1}, X^{2} \in \mathcal{K} .
$$

Proof: Expanding (16), we obtain:

$$
\begin{gathered}
\left\langle\left(F\left(X^{1}\right)-F\left(X^{2}\right)\right)^{T}, X^{1}-X^{2}\right\rangle \\
=\sum_{a \in L}\left[\left(\frac{\partial \hat{c}_{a}\left(f_{a}^{1}\right)}{\partial f_{a}}+\omega \frac{\partial e_{a}\left(f_{a}^{1}\right)}{\partial f_{a}}+\beta_{a}^{1}\right)-\left(\frac{\partial \hat{c}_{a}\left(f_{a}^{2}\right)}{\partial f_{a}}+\omega \frac{\partial e_{a}\left(f_{a}^{2}\right)}{\partial f_{a}}+\beta_{a}^{2}\right)\right] \times\left[f_{a}^{1}-f_{a}^{2}\right] \\
+\sum_{a \in L}\left[\left(\frac{\partial \hat{\pi}_{a}\left(u_{a}^{1}\right)}{u_{a}}+\omega \frac{\partial \hat{e}_{a}\left(u_{a}^{1}\right)}{\partial u_{a}}-\beta_{a}^{1}\right)-\left(\frac{\partial \hat{\pi}_{a}\left(u_{a}^{2}\right)}{\partial u_{a}}+\omega \frac{\partial \hat{e}_{a}\left(u_{a}^{2}\right)}{\partial u_{a}}-\beta_{a}^{2}\right)\right] \times\left[u_{a}^{1}-u_{a}^{2}\right] \\
+\sum_{a \in L}\left[\left(u_{a}^{1}-f_{a}^{1}\right)-\left(u_{a}^{2}-f_{a}^{2}\right)\right] \times\left[\beta_{a}^{1}-\beta_{a}^{2}\right] \\
=\sum_{a \in L}\left[\frac{\partial \hat{c}_{a}\left(f_{a}^{1}\right)}{\partial f_{a}}-\frac{\partial \hat{c}_{a}\left(f_{a}^{2}\right)}{\partial f_{a}^{2}}\right] \times\left[f_{a}^{1}-f_{a}^{2}\right]+\omega \sum_{a \in L}\left[\frac{\partial e_{a}\left(f_{a}^{1}\right)}{\partial f_{a}}-\frac{\partial e_{a}\left(f_{a}^{2}\right)}{\partial f_{a}^{2}}\right] \times\left[f_{a}^{1}-f_{a}^{2}\right] \\
+\sum_{a \in L}\left[\frac{\partial \hat{\pi}_{a}\left(u_{a}^{1}\right)}{\partial u_{a}}-\frac{\partial \hat{\pi}_{a}\left(u_{a}^{2}\right)}{\partial u_{a}}\right] \times\left[u_{a}^{1}-u_{a}^{2}\right]+\omega \sum_{a \in L}\left[\frac{\partial \hat{e}_{a}\left(u_{a}^{1}\right)}{\partial u_{a}}-\frac{\partial \hat{e}_{a}\left(u_{a}^{2}\right)}{\partial u_{a}}\right] \times\left[u_{a}^{1}-u_{a}^{2}\right] .
\end{gathered}
$$

But the expression in (17) is greater than or equal to zero, since we have assumed that both the total cost and the emission functions are convex and continuously differentiable and that the weight $\omega$ is nonnegative. Hence, the result has been established.

\section{Theorem 3}

The function $F(X)$ as defined following (12) is Lipschitz continuous, that is,

$$
\left\|F\left(X^{1}\right)-F\left(X^{2}\right)\right\| \leq\left\|X^{1}-X^{2}\right\|, \quad \forall X^{1}, X^{2} \in \mathcal{K} .
$$

Proof: Since we have assumed that the $\hat{c}_{a}\left(f_{a}\right)$ functions, the $\hat{\pi}_{a}\left(u_{a}\right)$, the $e_{a}\left(f_{a}\right)$ and the $\hat{e}_{a}\left(u_{a}\right)$ functions all have bounded second-order derivatives for all links $a \in L$, the result 
is direct by applying a mid-value theorem from calculus to the function $F$ that enters the above variational inequality.

We now state the convergence result for the modified projection method for this model.

\section{Theorem 4: Convergence}

Assume that the function that enters the variational inequality (11) (or (12)) has at least one solution and satisfies the conditions in Theorem 2 and in Theorem 3. Then the modified projection method converges to the solution of the variational inequality (11) (or (12)).

Proof: According to Korpelevich (1977), the modified projection method converges to the solution of the variational inequality problem of the form (12), provided that a solution exists and that the function $F$ that enters the variational inequality is monotone and Lipschitz continuous and that a solution exists. Monotonicity follows from Theorem 2. Lipschitz continuity, in turn, follows from Theorem 3.

The subproblems induced by the modified projection method correspond to separable, quadratic optimization problems in product flows, link capacities, and Lagrange multipliers, respectively. The quadratic programming problem in product flows corresponds to the classical system - optimization problem (cf. Dafermos and Sparrow (1969) and Nagurney (1999)), for which numerous efficient algorithms exist since transportation network problems are widely solved in practice. The solutions to the link capacity subproblems, as well as the Lagrange multiplier subproblems, in turn, can be obtained via closed form expressions, since the underlying feasible sets are very simple. Indeed, in the case of the sustainable supply chain network design problem, for example, the latter consist exclusively of nonnegativity constraints.

We would like to emphasize that the design of sustainable supply chain networks may also be viewed as a class of problems related to the broader set of problems in network design, but with specific features. For an excellent overview of optimization problems, including network design problems, faced in the context of telecommunications (and transportation), see Migdalas (2006). In this paper, we focus on the design of sustainable supply chain networks and the variational inequality formulation that is particularly suited for analysis 
and computations. Furthermore, this sustainable supply chain network design model, and accompanying variational inequality formulation, can be used as the basic framework for the development of many extensions and applications. For example, a possible extension would be to capture competition for resources (as well as sharing of resources, such as distribution centers) among different firms in complex supply chains. These might include, for example, firms engaged in oligopolistic competition, who seek to determine their individual supply chain network designs (and the associated environmental impacts). In addition, one might consider also the design of sustainable multiproduct supply chain networks.

\section{Numerical Examples}

The modified projected method was implemented in FORTRAN and a Unix system at the University of Massachusetts Amherst was used for all the computations. We initialized the algorithm by equally distributing the demand at each demand site among all the paths joining the firm node 0 to the demand node. All other variables, that is, the link capacities and the Lagrange multipliers, were initialized to zero.

The baseline supply chain network topology for all the examples is as depicted in Figure 2 with the links defined by numbers as in Figure 2. The numerical examples, hence, consisted of a firm faced with 3 possible manufacturing plants, each of which had 2 possible technologies, 2 distribution centers, each of which also had 2 distinct technologies, and the firm had to supply the 3 demand points. There was only a single mode of transportation/shipment available between each manufacturing plant and each distribution center and between each distribution center at a given demand point.

The common input data for the three examples is reported in Table 1. 


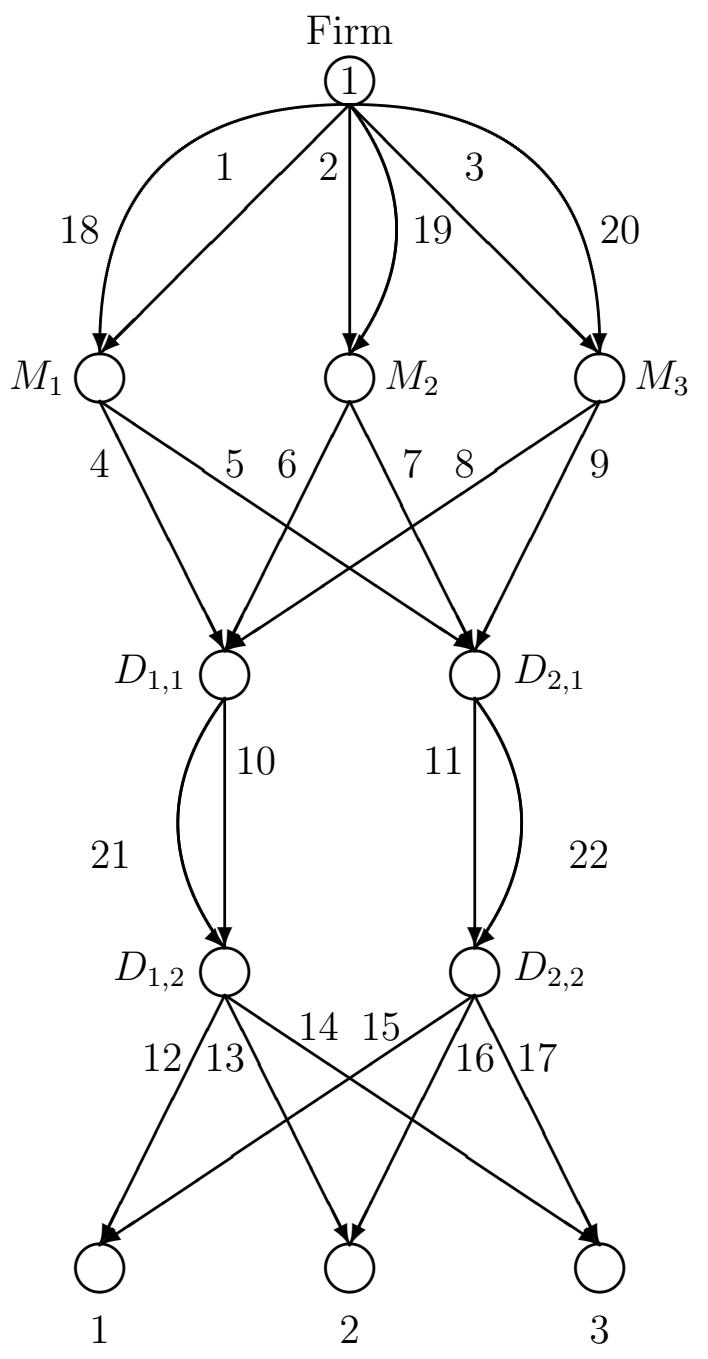

Figure 2: The Baseline Supply Chain Network Topology $G=[N, L]$ for the Examples 
Table 1: Total Cost and Emission Functions for the Numerical Examples

\begin{tabular}{|c|c|c|c|c|}
\hline Link $a$ & $\hat{c}_{a}\left(f_{a}\right)$ & $\hat{\pi}_{a}\left(u_{a}\right)$ & $e_{a}\left(f_{a}\right)$ & $\hat{e}_{a}\left(u_{a}\right)$ \\
\hline 1 & $f_{1}^{2}+2 f_{1}$ & $.5 u_{1}^{2}+u_{1}$ & $.05 f_{1}^{2}+f_{1}$ & $1.5 u_{1}^{2}+2 u_{1}$ \\
\hline 2 & $.5 f_{2}^{2}+f_{2}$ & $2.5 u_{2}^{2}+u_{2}$ & $.1 f_{2}^{2}+f_{2}$ & $2 u_{2}^{2}+2 u_{2}$ \\
\hline 3 & $.5 f_{3}^{2}+f_{3}$ & $u_{3}^{2}+2 u_{3}$ & $.15 f_{3}^{2}+2 f_{3}$ & $2.5 u_{3}^{2}+u_{3}$ \\
\hline 4 & $1.5 f_{4}^{2}+2 f_{4}$ & $u_{4}^{2}+u_{4}$ & $.05 f_{4}^{2}+.1 f_{4}$ & $.1 u_{4}^{2}+.2 u_{4}$ \\
\hline 5 & $f_{5}^{2}+3 f_{5}$ & $2.5 u_{5}^{2}+2 u_{5}$ & $.05 f_{5}^{2}+.1 f_{5}$ & $.05 u_{5}^{2}+.1 u_{5}$ \\
\hline 6 & $f_{6}^{2}+2 f_{6}$ & $.5 u_{6}^{2}+u_{6}$ & $.1 f_{6}^{2}+.1 f_{6}$ & $.05 u_{6}^{2}+.1 u_{6}$ \\
\hline 7 & $.5 f_{7}^{2}+2 f_{7}$ & $.5 u_{7}^{2}+u_{7}$ & $.05 f_{7}^{2}+.2 f_{7}$ & $.1 u_{7}^{2}+.2 u_{7}$ \\
\hline 8 & $.5 f_{8}^{2}+2 f_{8}$ & $1.5 u_{8}^{2}+u_{8}$ & $.05 f_{8}^{2}+.1 f_{8}$ & $.1 u_{8}^{2}+.3 u_{8}$ \\
\hline 9 & $f_{9}^{2}+5 f_{9}$ & $2 u_{9}^{2}+3 u_{9}$ & $.05 f_{9}^{2}+.1 f_{9}$ & $.1 u_{9}^{2}+.2 u_{9}$ \\
\hline 10 & $.5 f_{10}^{2}+2 f_{10}$ & $u_{10}^{2}+5 u_{10}$ & $.2 f_{10}^{2}+f_{10}$ & $1.5 u_{10}^{2}+3 u_{10}$ \\
\hline 11 & $f_{11}^{2}+f_{11}$ & $.5 u_{11}^{2}+3 u_{11}$ & $.25 f_{11}^{2}+3 f_{11}$ & $2 u_{11}^{2}+3 u_{11}$ \\
\hline 12 & $.5 f_{12}^{2}+2 f_{12}$ & $.5 u_{12}^{2}+u_{12}$ & $.05 f_{12}^{2}+.1 f_{12}$ & $.1 u_{12}^{2}+.2 u_{12}$ \\
\hline 13 & $.5 f_{13}^{2}+5 f_{13}$ & $.5 u_{13}^{2}+u_{13}$ & $.1 f_{13}^{2}+.1 f_{13}$ & $.05 u_{13}^{2}+.1 u_{13}$ \\
\hline 14 & $f_{14}^{2}+7 f_{14}$ & $2 u_{14}^{2}+5 u_{14}$ & $.15 f_{14}^{2}+.2 f_{14}$ & $.1 u_{14}^{2}+.1 u_{14}$ \\
\hline 15 & $f_{15}^{2}+2 f_{15}$ & $.5 u_{15}^{2}+u_{15}$ & $.05 f_{15}^{2}+.3 f_{15}$ & $.1 u_{15}^{2}+.2 u_{15}$ \\
\hline 16 & $.5 f_{16}^{2}+3 f_{16}$ & $u_{16}^{2}+u_{16}$ & $.05 f_{16}^{2}+.1 f_{16}$ & $.1 u_{16}^{2}+.1 u_{16}$ \\
\hline 17 & $.5 f_{17}^{2}+2 f_{17}$ & $.5 u_{17}^{2}+u_{17}$ & $.15 f_{17}^{2}+.3 f_{17}$ & $.05 u_{17}^{2}+.1 u_{17}$ \\
\hline 18 & $.5 f_{18}^{2}+f_{18}$ & $u_{18}^{2}+2 u_{18}$ & $.2 f_{18}^{2}+2 f_{18}$ & $2 u_{18}^{2}+3 u_{18}$ \\
\hline 19 & $.5 f_{19}^{2}+2 f_{19}$ & $u_{19}^{2}+u_{19}$ & $.25 f_{19}^{2}+3 f_{19}$ & $3 u_{19}^{2}+4 u_{19}$ \\
\hline 20 & $1.5 f_{20}^{2}+f_{20}$ & $u_{20}^{2}+u_{20}$ & $.3 f_{20}^{2}+3 f_{20}$ & $2.5 u_{20}^{2}+5 u_{20}$ \\
\hline 21 & $.5 f_{21}^{2}+2 f_{21}$ & $u_{21}^{2}+3 u_{21}$ & $1 f_{21}^{2}+3 f_{21}$ & $1.5 u_{21}^{2}+4 u_{21}$ \\
\hline 22 & $f_{22}^{2}+3 f_{22}$ & $.5 u_{22}^{2}+2 u_{22}$ & $.2 f_{22}^{2}+4 f_{22}$ & $2.5 u_{22}^{2}+4 u_{22}$ \\
\hline
\end{tabular}




\section{Example 1}

In Example 1 the demands were:

$$
d_{1}=45, \quad d_{2}=35, \quad d_{3}=5
$$

The total cost and emission functions were as reported in Table 1. In Example 1 we assumed that the firm did not care about the emissions generated in its supply chain design and, hence, $\omega=0$. The computed solution is reported in Table 2 . The total cost (see objective function (6)) was: 10,716.33. The total emissions (see objective function (7)) generated were: $8,630.45$. The value of the objective function (8) was, hence, 10,716.33. Note that link 14 has zero capacity and, thus, zero flow. Hence, the final optimal sustainable supply chain network topology for this problem is the network in Figure 2 but with link 14 removed.

\section{Example 2}

Example 2 had the identical data as in Example 1 except that the firm now was more concerned about the environment with $\omega=5$. The new computed solution is given in Table 3. The total cost (see objective function (6)) was now: 11,285.04. The total emissions (see objective function (7)) generated were now: 7,759.35. The value of the objective function (8) was, hence, 50,081.77. Due to the higher weight on the total emissions generated, the number of emissions decreased relative to the amount generated in Example 1. However, as a consequence, the total cost was now higher than in Example 1. Note that now all the links have positive capacity and positive flows. Hence, the final optimal supply chain network topology for this example is that in Figure 2.

Observe that whereas links 1 and 18 had the same product flows in Example 1, in Example 2, the production shifted from link 18 to link 1 at about a $50 \%$ increase, since link 1 corresponded to more environmentally-friendly technology. Similar behavior, occurred with links 2 and 19. 
Table 2: Example 1 Solution

\begin{tabular}{|c|c|c|c|}
\hline Link $a$ & $f_{a}^{*}$ & $u_{a}^{*}$ & $\beta_{a}^{*}$ \\
\hline 1 & 12.43 & 12.43 & 13.43 \\
\hline 2 & 11.67 & 11.67 & 59.33 \\
\hline 3 & 15.81 & 15.81 & 33.62 \\
\hline 4 & 14.69 & 14.69 & 30.39 \\
\hline 5 & 10.16 & 10.16 & 52.82 \\
\hline 6 & 13.94 & 13.94 & 14.94 \\
\hline 7 & 20.70 & 20.70 & 12.70 \\
\hline 8 & 15.83 & 15.83 & 48.50 \\
\hline 9 & 9.66 & 9.66 & 41.66 \\
\hline 10 & 21.90 & 21.90 & 48.80 \\
\hline 11 & 20.43 & 20.43 & 23.43 \\
\hline 12 & 25.44 & 25.44 & 26.44 \\
\hline 13 & 19.03 & 19.03 & 20.03 \\
\hline 14 & 0.00 & 0.00 & 3.85 \\
\hline 15 & 19.56 & 19.56 & 20.56 \\
\hline 16 & 15.97 & 15.97 & 32.93 \\
\hline 17 & 5.00 & 5.00 & 6.00 \\
\hline 18 & 12.43 & 12.43 & 26.86 \\
\hline 19 & 22.98 & 22.98 & 46.95 \\
\hline 20 & 9.69 & 9.69 & 20.37 \\
\hline 21 & 22.57 & 22.57 & 48.14 \\
\hline 22 & 20.10 & 20.10 & 22.10 \\
\hline & & & \\
\hline
\end{tabular}


Table 3: Example 2 Solution

\begin{tabular}{|c|c|c|c|}
\hline Link $a$ & $f_{a}^{*}$ & $u_{a}^{*}$ & $\beta_{a}^{*}$ \\
\hline 1 & 19.32 & 19.32 & 320.17 \\
\hline 2 & 15.69 & 15.69 & 403.10 \\
\hline 3 & 13.45 & 13.45 & 370.17 \\
\hline 4 & 19.43 & 19.43 & 60.29 \\
\hline 5 & 13.80 & 13.80 & 78.38 \\
\hline 6 & 13.75 & 13.75 & 22.12 \\
\hline 7 & 13.28 & 13.28 & 28.55 \\
\hline 8 & 15.73 & 15.73 & 65.43 \\
\hline 9 & 9.02 & 9.02 & 49.09 \\
\hline 10 & 24.03 & 24.03 & 428.53 \\
\hline 11 & 19.71 & 19.71 & 431.81 \\
\hline 12 & 26.62 & 26.62 & 55.23 \\
\hline 13 & 20.62 & 20.62 & 32.43 \\
\hline 14 & 1.67 & 1.67 & 13.87 \\
\hline 15 & 18.38 & 18.38 & 38.77 \\
\hline 16 & 14.38 & 14.38 & 44.65 \\
\hline 17 & 3.33 & 3.33 & 6.49 \\
\hline 18 & 13.90 & 13.90 & 322.79 \\
\hline 19 & 11.34 & 11.34 & 383.79 \\
\hline 20 & 11.30 & 11.30 & 331.01 \\
\hline 21 & 24.88 & 24.88 & 445.88 \\
\hline 22 & 16.38 & 16.38 & 447.95 \\
\hline & & & \\
\hline
\end{tabular}


Table 4: Example 3 Solution

\begin{tabular}{|c|c|c|c|}
\hline Link $a$ & $f_{a}^{*}$ & $u_{a}^{*}$ & $\beta_{a}^{*}$ \\
\hline 1 & 20.16 & 20.16 & 645.86 \\
\hline 2 & 15.80 & 15.80 & 731.98 \\
\hline 3 & 13.10 & 13.10 & 693.10 \\
\hline 4 & 19.66 & 19.66 & 81.62 \\
\hline 5 & 14.66 & 14.66 & 90.97 \\
\hline 6 & 14.37 & 14.37 & 30.74 \\
\hline 7 & 11.99 & 11.99 & 38.96 \\
\hline 8 & 15.45 & 15.45 & 81.25 \\
\hline 9 & 8.88 & 8.88 & 58.25 \\
\hline 10 & 24.30 & 24.30 & 812.50 \\
\hline 11 & 19.49 & 19.49 & 831.78 \\
\hline 12 & 26.44 & 26.44 & 82.33 \\
\hline 13 & 20.63 & 20.63 & 43.25 \\
\hline 14 & 2.40 & 2.40 & 20.43 \\
\hline 15 & 18.56 & 18.56 & 58.67 \\
\hline 16 & 14.37 & 14.37 & 59.50 \\
\hline 17 & 2.60 & 2.60 & 7.19 \\
\hline 18 & 14.16 & 14.16 & 626.55 \\
\hline 19 & 10.55 & 10.55 & 695.13 \\
\hline 20 & 11.22 & 11.22 & 634.49 \\
\hline 21 & 25.17 & 25.17 & 848.48 \\
\hline 22 & 16.04 & 16.04 & 859.93 \\
\hline & & &
\end{tabular}

\section{Example 3}

Example 3 had the same data as Examples 1 and 2 but now the firm was even more concerned about the environment with $\omega=10$. The new solution is given in Table 4 . The total cost was: 11,414.07. The total emissions generated were now: 7,739.32. The value of the objective function (8) was 88,807.30. As in Example 2, all links have positive capacity and positive product flow at the optimal solution.

The above examples, although stylized, illustrate the practicality and flexibility of the modeling approach and algorithm. 


\section{Summary and Conclusions}

In this paper, we developed a sustainable supply chain network design model that allows for the evaluation of environmental multicriteria decision-making. The model consists of two objective functions: total cost minimization, in which the total cost includes both the capital cost associated with link formation as well as the operational cost over the time horizon of interest, and the total emissions generated. A weighted objective function is then constructed, which includes the weight that the firm places on the minimization of emission generation. The variables in the optimization model include the link capacities associated with the manufacturing plants, the distribution centers, and the shipment modes, each of which can also be evaluated in terms of distinct technologies (and, hence, distinct emissions), and the product flows on the supply chain network links.

We constructed the optimization model and established its variational inequality formulation along with some qualitative results. An algorithm was then proposed for the solution of the variational inequality, along with convergence results. Finally, we applied the algorithm to several numerical sustainable supply chain network design problems for which the optimal capacities, the optimal product flows, and the associated optimal Lagrange multipliers were computed, when the weight associated with the environmental criterion was varied.

Using the formalism described in this paper, a firm may engineer its supply chain to be not only fiscally cost effective, but also environmentally responsible.

\section{Acknowledgments}

The authors acknowledge the constructive comments and suggestions of two anonymous reviewers and of the Editor on an earlier version of this manuscript.

The first author's research was supported by the John F. Smith Memorial Fund at the Isenberg School of Management. The second author acknowledges support from the University of Hartford while he was on sabbatical leave at the NSF ERC Center CASA at the University of Massachusetts Amherst. The financial support is gratefully acknowledged. 


\section{References}

Bertsekas, D.P., Tsitsiklis, J.N., 1989. Parallel and distributed computation - Numerical methods. Prentice Hall, Englewood Cliffs, New Jersey.

Bloemhof-Ruwaard, J.M., Beek, P.V., Hordijk, L. and Van Wassenhove, L., 1995. Interactions between operational research and environmental management. European Journal of Operational Research, 85, 229-243.

Dafermos, S.C. and Sparrow, F.T., 1969. The traffic assignment problem for a general network. Journal of Research of the National Bureau of Standards, 73B, 91-118.

Dhanda, K.K., Nagurney, A. and Ramanujam, P., 1999. Environmental networks: A framework for economic decision-making. Edward Elgar Publishing, Cheltenham, England.

Fabian, T., 2000. Supply chain management in an era of social and environment accountability. Sustainable Development International, 2, 27-30.

Hill, K.E., 1997. Supply-chain dynamics, environmental issues, and manufacturing firms. Environment \&f Planning A, 29, 1257-1274.

Ingram, M., 2002. Producing the natural fiber naturally: Technological change and the US organic cotton industry. Agriculture and Human Values, 19, 325-336.

Korpelevich, G.M., 1977. The extragradient method for finding saddle points and other problems. Matekon, 13, 35-49.

Migdalas, A., 2006. Nonlinear programming in telecommunications. In: M.G.C. Resende and P.M. Pardalos, eds. Handbook of optimization. Springer, New York, pp. 27-57.

Nagurney, A., 1999. Network economics: A variational inequality approach, second and revised edition. Kluwer Academic Publishers, Dordrecht, The Netherlands.

Nagurney, A., 2006. Supply chain network economics: Dynamics of prices, flows and profits. Edward Elgar Publishing, Cheltenham, England. 
Nagurney, A., Cruz, J. and Matsypura, D., 2003. Dynamics of global supply chain supernetworks. Mathematical and Computer Modelling, 37, 963-983.

Nagurney, A., Dong, J. and Zhang, D., 2002. A supply chain network equilibrium model. Transportation Research E, 38, 281-303.

Nagurney, A., Liu, Z. and Woolley, T., 2007. Sustainable supply chain networks and transportation. International Journal of Sustainable Transportation, 1, 29-51.

Nagurney, A., Qiang, Q. and Nagurney, L. S., 2010. Environmental impact assessment of transportation networks with degradable links in an era of climate change. International Journal of Sustainable Transportation 4, 154-171.

Nagurney, A. and Toyasaki, F., 2005. Reverse supply chain management and electronic waste recycling: A multitiered network equilibrium framework for e-cycling. Transportation Research E, 41, 1-28.

Nagurney, A. and Woolley, T., 2010. Environmental and cost synergy in supply chain network integration in mergers and acquisitions. In: M. Ehrgott, B., Naujoks, T. Stewart and J. Wallenius, eds. Sustainable energy and transportation systems, Proceedings of the 19th International Conference on Multiple Criteria Decision Making, Lecture notes in economics and mathematical systems. Springer, Berlin, Germany, 51-78.

Qiang, Q. and Nagurney, A. and Dong, J. 2009. Modeling of supply chain risk under disruptions with performance measurement and robustness analysis. In: T. Wu and J. Blackhurst, eds. Managing supply chain risk and vulnerability: Tools and methods for supply chain decision makers. Springer, Berlin, Germany, 91-111.

Rahimifard, S. and Clegg, A. J., 2007. Aspects of sustainable design and manufacture. International Journal of Production Research, 45, 4013-4019.

Wu, K., Nagurney, A., Liu, Z. and Stranlund, J.K., 2006. Modelling generator power plant portfolios and pollution taxes in electric power supply chain networks: A transportation network equilibrium transformation. Transportation Research D, 11, 171-190. 